\title{
Expansion of an interacting Fermi gas
}

\author{
${ }^{1,2} \mathrm{C}$. Menotti, ${ }^{1} \mathrm{P}$. Pedri and ${ }^{1} \mathrm{~S}$. Stringari \\ 1 Dipartimento di Fisica, Università di Trento and BEC-INFM, \\ I-38050 Povo, Italy \\ 2 Dipartimento di Matematica e Fisica, Università Cattolica del Sacro Cuore, \\ I-25121 Brescia, Italy
}

(October 31, 2018)

We study the expansion of a dilute ultracold sample of fermions initially trapped in a anisotropic harmonic trap. The expansion of the cloud provides valuable information about the state of the system and the role of interactions. In particular the time evolution of the deformation of the expanding cloud behaves quite differently depending on whether the system is in the normal or in the superfluid phase. For the superfluid phase, we predict an inversion of the deformation of the sample, similarly to what happens with Bose-Einstein condensates. Viceversa, in the normal phase, the inversion of the aspect ratio is never achieved, if the mean field interaction is attractive and collisions are negligible.

Since the first experiments on trapped Bose-Einstein condensates the imaging of the expanding cloud, following the sudden switching off of the confining potential, has provided crucial information on the novel features exhibited by atomic gases in conditions of quantum degeneracy. These include, in particular, the bimodal structure of an expanding Bose gas at finite temperature and the anisotropy of the asymptotic profile of the condensate $[1,2]$. In the Thomas-Fermi limit, where the Gross-Pitaevskii equations coincide with the hydrodynamic theory of superfluids, the anisotropy of the expanded gas reflects the anisotropy of the pressure force which is stronger in the direction of tighter confinement [3]. The predictions of the hydrodynamic equations and of the consequent scaling behaviour exhibited by BoseEinstein condensates during the expansion have been investigated by several authors ( [4], [5], [6]), providing excellent agreement with experiments $[7,8]$ and pointing out the difference with respect to the expansion of a non condensed gas. In the latter case, in the collisionless regime, the density profile approaches an isotropic shape, independent of the initial deformation of the gas.

In this letter we study the problem of the expansion of an ultracold sample of fermions initially trapped in an anisotropic harmonic trap. We will show that also in the case of fermions the expansion of the gas provides valuable information about the state of the system and the role of interactions. We will consider a gas of atoms interacting with attractive forces. This is a natural requirement for the realization of Cooper-pairs and hence for the achievement of the superfluid phase [9]. Such interactions are naturally present in some fermionc species like ${ }^{6} \mathrm{Li}$ and can otherwise be obtained by changing the scattering length profiting of the presence of a Feshbach resonance.

The description of the expansion of a cold fermionic gas in the normal and superfluid phase requires different theoretical approaches. For the normal phase we use the formalism of the Landau-Vlasov equations, while in the superfluid phase we study the expansion using the hydrodynamic theory of superfluids.

We consider the case of two different fermionic states, hereafter called 1 and 2, initially confined in a harmonic trap. We assume that the two species are present in the same amount and feel the same trapping potential, so that the densities of the two species are equal at equilibrium as well as during the expansion: $n_{1}(\mathbf{r}, t)=$ $n_{2}(\mathbf{r}, t)=n(\mathbf{r}, t) / 2$. The trapping potential will be chosen of harmonic type

$$
V_{h o}=\frac{1}{2} m\left(\omega_{\perp}^{2} x^{2}+\omega_{\perp}^{2} y^{2}+\omega_{z}^{2} z^{2}\right)
$$

describing a cilyndrically simmetric trap with deformation $\lambda=\omega_{z} / \omega_{\perp}$. The interaction between the two fermionic species is fixed by the coupling constant $g=$ $4 \pi \hbar^{2} a / m$, where $a$ is the $s$-wave scattering length.

In this paper we will use the equation of state

$$
\mu_{\ell e}(n)=\frac{\hbar^{2}}{2 m}\left(3 \pi^{2} n\right)^{2 / 3}+\frac{1}{2} g n,
$$

to describe the uniform phase of the gas where the first term is the kinetic energy evaluated at zero temperaure while the second one is the interaction energy evaluated in the mean field approximation. Equation (2) neglects the effects of correlations which become important for large values of the scattering length and affect in a different way the equation of state of the normal and superfluid phase. The formalism developed in this paper can be easily generalized to include a more accurate description of the equation of state. It is however worth pointing out that, even using the same equation of state for the normal and superfluid phases, the expansion of the gas behaves quite differently in the two cases being described by different kinetic equations. In the presence of the external potential (1), the equilibrium condition in the local density approximation is determined by the equation

$$
\mu_{\ell e}(n)+V_{h o}(\mathbf{r})=\mu
$$


where $\mu$ is the chemical potential of the sample fixed by the normalization condition.

The relevant parameter characterizing the interaction in the fermionic system is the ratio

$$
\chi=\frac{E_{i n t}}{E_{h o}}
$$

between the interaction energy

$$
E_{\text {int }}=\frac{g}{4} \int n^{2}(\mathbf{r}) d^{3} r
$$

and the oscillator energy

$$
E_{h o}=\int V_{h o}(\mathbf{r}) n(\mathbf{r}) d^{3} r .
$$

In the perturbative regime the integrals $(5,6)$ can be evaluated using the non interacting density profile which, in Thomas-Femi approximation (3), takes the simple form

$$
n(\mathbf{r})=\frac{1}{3 \pi^{2}}\left(\frac{2 m}{\hbar^{2}}\right)^{3 / 2}\left[\mu-V_{h o}(\mathbf{r})\right]^{3 / 2}
$$

After integration of (5) and (6) one finds [10]

$$
\frac{E_{i n t}}{E_{h o}}=0.5 \frac{N^{1 / 6} a}{a_{h o}}=0.3 a k_{F}
$$

where $k_{F}=\left(3 \pi^{2} n(0)\right)^{1 / 3}$ is the Fermi momentum evaluated at the central value of the density. In order to go beyond the perturbative regime, one determines numerically the ground state density by minimizing the energy of the system

$$
\begin{aligned}
E & =\frac{3\left(3 \pi^{2}\right)^{2 / 3}}{5} \frac{\hbar^{2}}{2 m} \int n^{5 / 3}(\mathbf{r}) d^{3} r+ \\
& +\int V_{h o}(\mathbf{r}) n(\mathbf{r}) d^{3} r+\frac{g}{4} \int n^{2}(\mathbf{r}) d^{3} r
\end{aligned}
$$

One easily finds that the equilibrium value of the ratio $E_{\text {int }} / E_{h o}$ depends on the dimensionless combination $a N^{1 / 6} / a_{h o}$ also in the non perturbative regime [9], as reported in Fig.1. Using (9) one predicts that the compressibility of the gas becomes negative in the center of the trap if $|a| N^{1 / 6} / a_{h o}>0.61$. For large values of $|a|$ the resulting predictions should be however taken with care since the functional (9) ignores correlation effects beyond mean field.

Let us now discuss the expansion of a fermionic sample trapped in an elongated harmonic trap $(\lambda<1)$. We describe first the expansion of the normal fluid and afterwards the one of the superfluid. In the ideal case, using the semiclassical description, one finds that the ratio of the square radii evolves according to the classical law

$$
\frac{\left\langle r_{\perp}^{2}\right\rangle}{\left\langle z^{2}\right\rangle}=\frac{1+\omega_{\perp}^{2} t^{2}}{1+\omega_{z}^{2} t^{2}} \frac{\omega_{z}^{2}}{\omega_{\perp}^{2}}
$$

The ratio (10) approaches unity for large times, reflecting the isotropy of the momentum distribution. This result ignores the effects of collisions which are however expected to play a minor role at low temperature, due to Pauli blocking, unless the scattering length is very large.

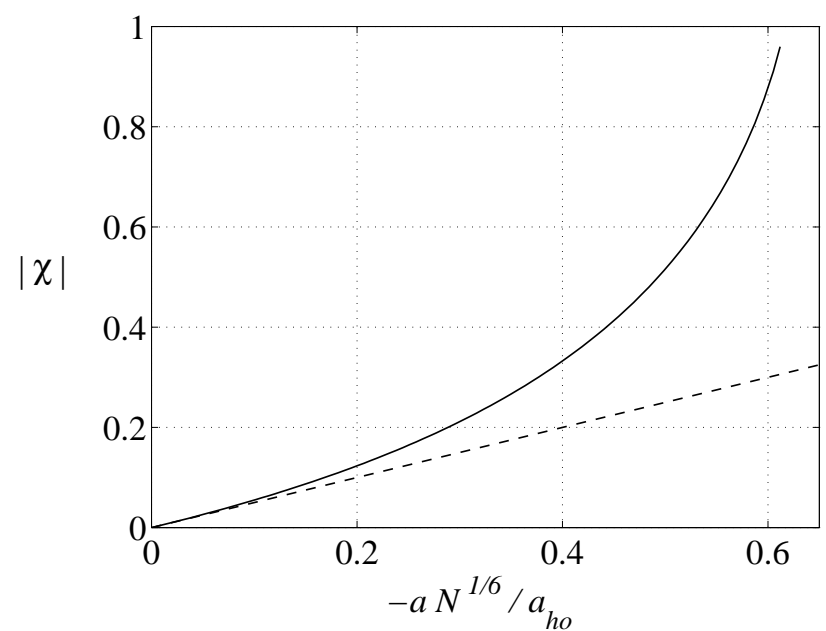

FIG. 1. Ratio $\chi=E_{\text {int }} / E_{\text {ho }}$ as a function of the universal parameter $a N^{1 / 6} / a_{h o}$ calculated with the mean field functional (9) up to the collapse point $a N^{1 / 6} / a_{h o}=-0.61$ (full line); the dashed line is the linear prediction (8).

In order to take into account the effects of the interactions, we consider the mean field description based on the Landau-Vlasov equation

$$
\frac{\partial f}{\partial t}+\mathbf{v} \cdot \frac{\partial f}{\partial \mathbf{r}}-\frac{\partial V_{h o}}{\partial \mathbf{r}} \cdot \frac{\partial f}{\partial \mathbf{v}}-\frac{1}{2} \frac{g}{m} \frac{\partial n}{\partial \mathbf{r}} \cdot \frac{\partial f}{\partial \mathbf{v}}=0
$$

where $f(\mathbf{r}, \mathbf{v}, t)$ is the distribution function, $n=\int f d^{3} v$ is the atomic density. Eq.(11) describes the dynamics of a normal weakly interacting gas in the collisionless regime.

An approximate solution of Eq.(11), is obtained by making a scaling ansatz for the distribution function

$$
f(\mathbf{r}, \mathbf{v}, t)=f_{0}(\tilde{\mathbf{r}}(t), \tilde{\mathbf{v}}(t))
$$

where $f_{0}$ is the equilibrium distribution, $\tilde{r}_{i}(t)=r_{i} / b_{i}$ and $\tilde{v}_{i}(t)=b_{i} v_{i}-\dot{b}_{i} r_{i}$. Under the scaling assumption the velocity field $\mathbf{u}(\mathbf{r}, t)=\int \mathbf{v} f d^{3} v / n$ takes the simple form $u_{i}=\dot{b}_{i} r_{i} / b_{i}$. This ansatz has been recently used by Guéry-Odelin [11] to investigate the effect of the interaction on the collective oscillation of a classical gas in the collisionless regime.

The equations for the scaling parameters $b_{i}$ can be obtained by multiplying (11) by $\tilde{r}_{i}$ and $\tilde{v}_{i}$ and integrating in phase space. Making use of the equilibrium properties of the distribution function, after some straigthforward algebra one finds

$$
\ddot{b}_{i}+\omega_{i}^{2} b_{i}-\frac{\omega_{i}^{2}}{b_{i}^{3}}+\frac{3}{2} \chi \omega_{i}^{2}\left(\frac{1}{b_{i}^{3}}-\frac{1}{b_{i} \prod_{j} b_{j}}\right)=0,
$$


where $\chi$ is the ratio (4) evaluated at equilibrium. The second term in (13) describes the restoring force of the external oscillator potential, the third one originates from the kinetic energy, while the last term, linear in $\chi$, accounts for the effects of mean field interaction.

An immediate application of Eq.(13) concerns the study of the oscillations of the gas. By linearizing the equations around equilibrium $\left(b_{i}=1\right)$ one finds, in the presence of isotropic harmonic trapping $\left(\omega_{\perp}=\omega_{z}=\omega_{0}\right)$, the result:

$$
\omega_{M}=2 \omega_{0} \sqrt{1+3 \chi / 8}, \omega_{Q}=2 \omega_{0} \sqrt{1-3 \chi / 4},
$$

for the frequencies of the monopole and quadrupole oscillations which coincide with the results already derived in [10] using a sum-rule approach.

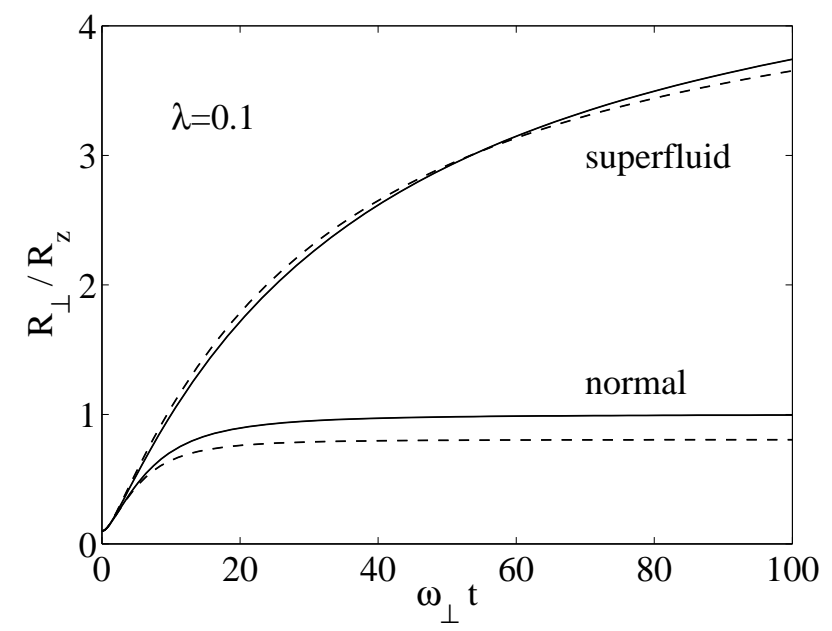

FIG. 2. Aspect ratio as a function of time for the expansion of the normal (lower curves) and superfluid phase (upper curves) for $\lambda=0.1$ and two different values of the parameter $\chi: \chi=0$ (full line) and $\chi=-0.4$ (dashed line).

The equations describing the expansion are obtained by suddenly removing the second term of Eq.(13), originating from the trapping potential. In the study of the expansion we are interested in the case of anisotropic trapping. In particular we will consider the case of cigar shaped traps. For high deformations $\left(\lambda=\omega_{z} / \omega_{\perp} \ll 1\right)$ Eq.(13) yields the asymptotic result

$$
\begin{aligned}
b_{z}^{2} & \rightarrow \omega_{z}^{2}\left(1-\frac{3}{2} \chi\right) t^{2}, \\
b_{\perp}^{2} & \rightarrow \omega_{\perp}^{2} t^{2},
\end{aligned}
$$

showing that the aspect ratio

$$
\frac{R_{\perp}(t)}{R_{z}(t)} \rightarrow \frac{1}{\sqrt{1-3 \chi / 2}}
$$

approaches a value smaller than 1 if the interaction is attractive $(\chi<0)$. In Eq.(17), $R_{\perp}$ and $R_{z}$ are the radii where the atomic density vanishes (Thomas-Fermi radii).
The results of the numeric integration of the equations of motion (13) are reported in Fig.2 and 3 as a function of time for the choices $\chi=0$ and $\chi=-0.4$.

We address now the problem of the expansion of a superfluid Fermi gas. As already anticipated we will make use of the hydrodynamic equations of superfluids. Those equations have been already used to describe the collective oscillations of a superluid trapped Fermi gas [12] including its rotational behaviour [13]. The hydrodynamic equations are applicable if the healing length is much smaller than the size of the sample, which implies that the energy gap should be larger than the oscillator energies $\hbar \omega_{z}, \hbar \omega_{\perp}$. This non trivial condition implies that the whole system behaves like superfluid. Furthermore the hydrodynamic equations are applicable up to excitation energies of the order of the energy gap. In the problem of the expansion it is crucial that the system remains superfluid in the first instants when the hydrodynamic forces provide the relevant acceleration to the expanding atoms. One expects that this condition be satisfied if the initial temperature is small enough.

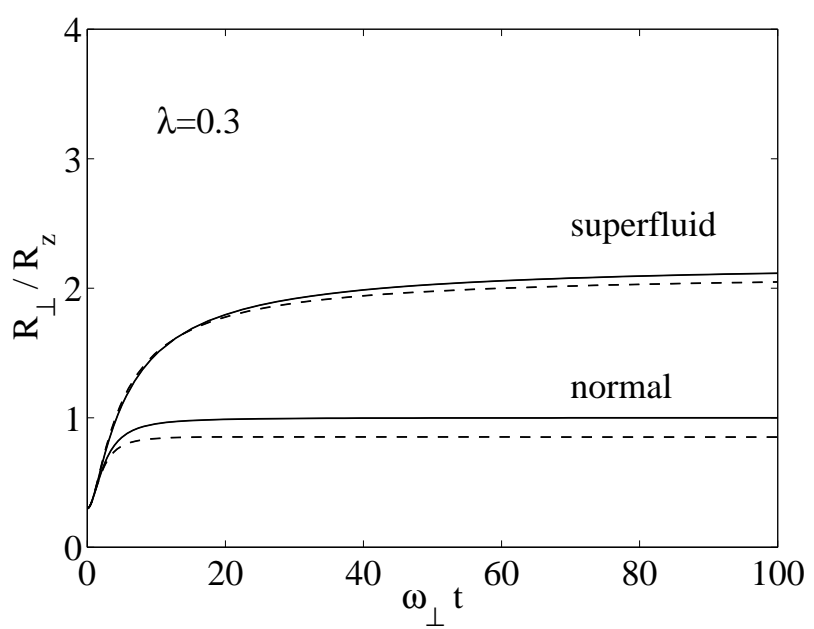

FIG. 3. Aspect ratio as a function of time for the expansion of the normal (lower curves) and superfluid phase (upper curves) for $\lambda=0.3$ and different values of the parameters $\chi$ : $\chi=0$ (full line) and $\chi=-0.4$ (dashed line).

The hydrodynamic description is based on the equation of continuity

$$
\frac{\partial}{\partial t} n+\nabla(n \mathbf{u})=0
$$

and on the Euler equation

$$
m \frac{\partial}{\partial t} \mathbf{u}+\nabla\left(\mu_{\ell e}(n)+V_{h o}(\mathbf{r})+\frac{1}{2} m u^{2}\right)=0
$$

where $\mu_{\ell e}(n)$ is the chemical potential of a uniform gas calculated at the density $n$ and $\mathbf{u}$ is the velocity field.

If the equation of state is a power law $\left(\mu_{\ell e} \propto n^{\gamma}\right)$ these equations admit the simple scaling solution 


$$
\begin{aligned}
n\left(r_{i}, t\right) & =\frac{1}{\prod_{j} b_{j}} n_{0}\left(\frac{r_{i}}{b_{i}}\right), \\
u_{i}\left(r_{i}, t\right) & =\frac{\dot{b}_{i}}{b_{i}} r_{i},
\end{aligned}
$$

and the Thomas-Fermi radii evolve according to the law $R_{i}(t)=R_{i}(0) b_{i}(t)$. In this case, it is immediate to show that, during the expansion, the scaling parameters obey the coupled differential equations

$$
\ddot{b}_{i}=\frac{\omega_{i}^{2}}{\left(b_{x} b_{y} b_{z}\right)^{\gamma} b_{i}} .
$$

which reduce to

$$
\ddot{b}_{z}=\frac{\omega_{z}^{2}}{b_{\perp}^{2 \gamma}}, \text { and } \ddot{b}_{\perp}=\frac{\omega_{\perp}^{2}}{b_{\perp}^{(2 \gamma+1)}}
$$

for highly elongated configurations $(\lambda \ll 1)$. For $\gamma=$ 1 (corresponding to a Bose-Einstein condensed gas) the equation for the radial motion is integrable analytically and one finds the result $b_{\perp}(t)=\left(1+\omega_{\perp}^{2} t^{2}\right)^{1 / 2}[5]$.

To describe the expansion of superfluid Fermi gas we will use the same equation of state (2) as for the normal phase. The case of a very dilute gas is also described by a power law with $\gamma=2 / 3$ (first term in (2)). For $\lambda=0.1$ and 0.3 the solution is given by the full upper line in Fig.2 and 3 respectively which show that the deformation of the trap is inverted in time and the aspect ratio $R_{\perp} / R_{z}$ reaches asymptotically a value significantly larger than 1 [14]. Superfluidity has hence the effect of distributing the release energy in a strongly asymmetric way along the axial and radial directions. It is worth noticing that the same scaling equations (22), with $\gamma=2 / 3$, are obtained for a classic gas in the collisional regime [15].

In the more general case (2), a useful approximation to the solution of the hydrodynamic equations, based on the scaling ansatz $(20,21)$, is obtained by multiplying the Euler's equation (19) by $r_{i} n(\mathbf{r})$ and integrating over the spatial coordinates. Using the equation of state (2), one finally obtains the following set of differential equations

$$
\begin{aligned}
& \ddot{b}_{i}+\omega_{i}^{2} b_{i}-\frac{\omega_{i}^{2}}{b_{i}} \frac{1}{\left(\prod_{i} b_{i}\right)^{2 / 3}}+ \\
& +\frac{3}{2} \chi \frac{\omega_{i}^{2}}{b_{i}}\left(\frac{1}{\left(\prod_{i} b_{i}\right)^{2 / 3}}-\frac{1}{\prod_{i} b_{i}}\right)=0,
\end{aligned}
$$

with $\chi$ defined by Eq.(4). Equations (24) differ from the anlalogous equations (13) holding in the normal phase. By linearizing Eqs.(24) around $b_{i}=1$ one gets, in the case of a spherical trap, the result $\omega_{Q}=\sqrt{2} \omega_{0}$ for the quadrupole frequency [12], which, contrary to (14), is independent of the interaction term in $\chi$.

The predictions of Eqs.(24) for the expansion of the gas are reported in Figs.2,3 and show that the inclusion of the interaction term in the equation of state affects the expansion of the superfluid only in a minor way.
In conclusion we have shown that the expansion of a superfluid Fermi gas, being governed by the equations of hydrodynamics, differs in a crucial way from the one of a normal gas in the collisionless regime. From a theoretical point of view several questions remain to be investigated: among them, the effect of large scattering lengths [16] on the equation of state and the role of collisions which, under certain conditions, might give rise to a hydrodynamic regime, and hence to anisotropic expansion, also in the normal phase. Finally one should develop the formalism at finite temperature where both the normal and superfluid components are present. The resulting bimodal structure in the expanding cloud is expected to be affected by the transfer of atoms from the superfluid to the normal component during the first stage of the expansion.

This research is supported by the Ministero dell'Istruzione, dell'Università e della Ricerca (MIUR). We acknowledge R. Combescot, G. Shlyapnikov and J. E. Thomas for useful discussions.

[1] M.H. Anderson, J.R. Ensher, M.R. Matthews, C.E. Wieman, and E.A. Cornell, Science 269, 198 (1995)

[2] K.B. Davis, M.-O. Mewes, M.R. Andrews, N.J. van Druten, D.S. Durfee, D.M. Kurn, and W. Ketterle, Phys. Rev. Lett. 75, 3969 (1995)

[3] F. Dalfovo, S. Giorgini, L.P. Pitaevskii and S. Stringari, Rev. Mod. Phys. 71, 463 (1999)

[4] Yu. Kagan, E.L. Surkov, and G.V. Shlyapnikov, Phys. Rev. A 54, R1753 (1996)

[5] Y. Castin and R. Dum, Phys. Rev. Lett. 77, 5315 (1996)

[6] F. Dalfovo, C. Minniti, and L.P. Pitaevskii, Phys. Rev. A 56, 4855-4863 (1997)

[7] D.M. Stamper-Kurn, M.R. Andrews, A.P. Chikkatur, S. Inouye, H.-J. Miessner, J. Stenger and W. Ketterle, Phys. Rev. Lett. 80, 2027 (1998)

[8] U. Ernst, J. Schuster, F. Schreck, A. Marte, A. Kuhn and G. Rempe, Appl. Phys. B 67, 719 (1998)

[9] H.T.C. Stoof, M. Houbiers, C.A. Sackett, and R.G. Hulet, Phys. Rev. Lett. 76, 10 (1996); M. Houbiers and H.T.C. Stoof, Phys. Rev. A 54, 5055 (1996)

[10] L. Vichi and S. Stringari, Phys. Rev. A 60, 4734 (1999)

[11] D. Guéry-Odelin, Phys. Rev. A 66, 033613 (2002)

[12] M.A. Baranov and D.S. Petrov, Phys. Rev. A 58, R801 (1998)

[13] A. Minguzzi and M.P. Tosi, Phys. Rev. A 63, 023609 (2001); F. Zambelli and S. Stringari, Phys. Rev. A 63, 033602 (2001)

[14] For highly elongated traps $(\lambda \ll 1)$, the asymptotic value of the aspect ratio is given by $0.38 / \lambda$, G.V. Shlyapnikov private communication

[15] Yu. Kagan, E.L. Surkov, and G.V. Shlyapnikov, Phys. Rev. A 55, R18 (1997)

[16] M.L. Chiofalo, S.J.J.M.F. Kokkelmans, J.N. Milstein and M.J. Holland, Phys. Rev. Lett. 88, 090402 (2002) 